\title{
Apparent arm length with active vs. passive touch
}

\author{
JERRY A. SCHLATER \\ Worcester Area Community Mental Health Center, Worcester, Massachusetts 01609 \\ A. HARVEY BAKER \\ Queens College, City University of New York, New York 10021
}

and

\author{
SEYMOUR WAPNER \\ Clark University, Worcester, Massachusetts 01610
}

\begin{abstract}
Two experiments tested the hypothesis that apparent length of the partially outstretched arm (1) shrinks when the fingertips of the passive hand are touched by an object and (2) increases when the fingertips of the hand touch an external, stationary object. Experiment 1 involved nominal judgments regarding which of two outstretched arms, each involved in a different type of contact, was longer. Experiment 2 used a single outstretched arm and involved interval scale measurements. Results, which supported the hypothesis, were interpreted as reflecting effects of the directionality of action (away from the person who is actively directed toward touching an object vs. toward the person who is passive and being touched) involved in the hand-object contact.
\end{abstract}

Earlier studies found that apparent extension of the outstretched arm (1) decreased when the fingertip was touched (Humphries, 1959) and (2) was judged to be shorter in a close-confined environmental context than in an open-extended context (Wapner, McFarland, \& Werner, 1963). These results were interpreted to indicate that experience of the boundary of the body was made more distinct by touching it or by placing the body so that the fingertips of the outstretched arm were close to a confined environmental context (viz., a wall). Increased articulation of the boundary presumably made for (1) an experience of increased separation of the body from context and (2) a decrease in perceived size of the body part (Wapner, 1969; Werner, 1940; see also Wapner, Werner, \& Comalli, 1958; Werner, Wapner, \& Comalli, 1957, for evidence on decrease in apparent head width with articulation of boundary through touch, etc.).

The process underlying the effect observed by Humphries (1959) may be reinterpreted. In Humphries' procedures, the experimenter touched the fingertips of the subject's stationary outstretched arm and hand. The finding of a decrease in apparent arm length with touch was interpreted as reflecting presence or absence

This investigation was supported in part by the United States Public Health Service Grant MH 00348 from the National Institute of Mental Health. The authors wish to thank Leonard Cirillo for his many insightful comments on an earlier draft of this paper and Irene W. Kostin for her valuable critical comments and editorial assistance in preparing this manuscript. of contact (touch vs. no touch). Touch, however, does not simply involve contact. Concurrent with the contact provided by touch is the directionality of the action (viz., whether the subject touched the experimenter or the experimenter touched the subject). These two variables (presence or absence of contact and directionality of action) were confounded in Humphries' study. "Being touched" could cause perceived shrinkage in arm length (which Humphries observed) and "touching by subject" could cause a perceived increase in arm length (an effect not hitherto observed).

\section{EXPERIMENT 1}

To explore the role of directionality of action in touching, conditions involving arm-hand movement had to be contrasted with the stationary arm-hand used by Humphries (1959). Such introduction of movement leads to a new problem with respect to proprioceptive activity. Being touched always involves "no movement," and touching always involves movement. Presence vs. absence of proprioceptive activity involved in movement results in variation in judged size of body part (Fuhrer \& Cowan, 1967). Experiment 1 was designed (cf. "Discussion," below) so that three of its six conditions provided a test of the directionality-of-action hypothesis compared with a proprioceptive motion hypothesis.

\section{Method}

Subject, blindfolded, with eyes closed, stood in a dark room with both arms partially outstretched and with only the spread- 


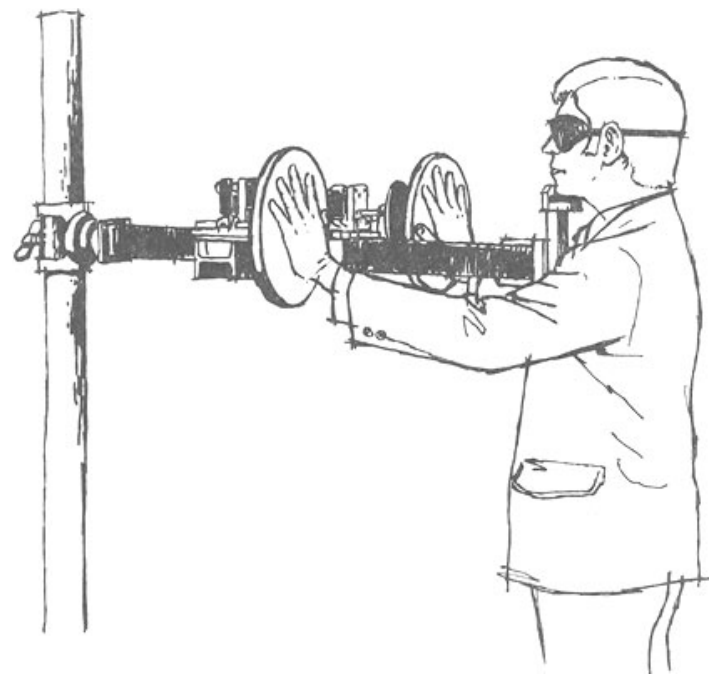

Figure 1. Subject positioned at the apparatus in Experiment 1.

apart fingertips touching two textured disks mounted in the frontoparallel plane (Figure 1). Each disk could be independently oscillated clockwise (CW) and counterclockwise (CCW) $35 \mathrm{times} / \mathrm{min}$, covering a radial distance of $42 \mathrm{deg}$. The two disks, each $25.4 \mathrm{~cm}$ in diameter, were covered with aluminum screening to provide heightened tactual experience. The $\mathrm{CW} / \mathrm{CCW}$ oscillating motion of each disk was accomplished by having it attached eccentrically to a smaller disk driven by a 35-rpm gear motor.

The task was to indicate which arm was judged as longer, and arm length was defined for the subject as the shortest distance from fingertips to shoulder. During this task, each hand was simultaneously engaged in one of four different types of tactual contact with a disk: In passive touch (A), the disk oscillated over the subject's stationary fingertips (directionality of action here was from moving object toward stationary subject). In active touch (B), the hand and fingertips oscillated over the surface of the stationary disk, in approximately the same arc and at approximately the same speed as the disk oscillated in passive touch (Directionality of action was from hand to stationary object). The two control conditions were control/ stationary $(\mathrm{C})$, in which both hand and disk were stationary, and control/moving (D), in which both hand and disk oscillated $\mathrm{CW}$ and $\mathrm{CCW}$ in the same direction at the same rate of speed, so that the subject's fingertips, while moving, remained at the same points on the surface of the disk. (No differential directionality was involved in Conditions $\mathrm{C}$ and $\mathrm{D}$.)

Each type of tactual contact was practiced for about 2 min until the experimenter was satisfied that the subject could perform adequately. In each type of contact, approximately identical sensory receptors of fingertips were in contact with the textured surfaces, a feature not always employed in studies involving active vs. passive touch (Gibson, 1962; Frey, Note 1; Frey \& Craven, Note 2), in which, for example, fingertips are used for active and palms for passive touch.

The four types of contact (A, B, C, D) were combined two at a time (one for each hand), making six conditions ( $\mathrm{AB}, \mathrm{AC}$, $\mathrm{AD}, \mathrm{BC}, \mathrm{BD}, \mathrm{CD})$. There were four consecutive trials for each condition, with sequence of condition systematically varied in a Latin square design (two subjects in each sequence). Within the four trials of each condition, there was equal representation of right and left hands for each type of contact.

The measure recorded was the arm the subject judged as longer. It was expected that (1) the arm involved in either of the two types of control contact would be experienced as shorter than the arm involved in active touch and longer than the arm involved in passive touch and (2) the active touch arm would be experienced as longer than the arm with passive touch. Although no differences were expected between the control/ stationary and control/moving conditions, for purposes of scoring, control/moving was arbitrarily treated as if it were predicted as longer. The percentage of trials that each subject's response was in the predicted direction served as the subject's score for a given condition (e.g., a score of $75 \%$ for the active touch/passive touch condition indicates that on three of the four trials, the subject reported that he judged his arm under active touch as longer).

Since each of the six conditions involved a comparison between two different types of contact, each condition was analyzed separately with a $t$ test, comparing the mean of each condition with a mean of $50 \%$, the outcome that would be expected on the basis of chance according to the scoring system.

Experiment 1 was conducted on two occasions. Twelve male college students served as subjects in Experiment $1 \mathrm{~A}$ and another 12 in Experiment 1B.

\section{Results}

The findings (Experiments $1 \mathrm{~A}$ and $1 \mathrm{~B})$ generally fit with expectation: Condition $\mathrm{AB}-$ When one hand is in active touch with the disk and the other in passiv touch, the arm with active touch is judged as extending longer [for Experiments $1 \mathrm{~A}$ and 1B, respectively, means $=77 \%$ and $75 \%, \mathrm{SDs}=17 \%$ and $15 \%$; ts $(11)=5.4$ and 5.9 , each $\mathrm{p}<.01$ (one-tailed test)]. Condition AD-When active touch was compared with control/moving, arm extension was longer with active touch [means $=75 \%$ and $71 \%, \mathrm{SDs}=18 \%$ and $21 \%$; $\operatorname{ts}(11)=4.7$ and 3.7 ; each $\mathrm{p}<.01$ ]. Conditions $\mathrm{BC}$ and $\mathrm{BD}-$ When either of the two control types of contact was compared with passive touch, judged arm extension was longer with the control type of contact (for control/moving: means = $79 \%$ and $79 \%, \mathrm{SDs}=18 \%$ and $14 \% ; \operatorname{ts}(11)=5.6$ and 6.9 , each $\mathrm{p}<.01$; for control/stationary: mean $=87 \%$ and $83 \%$, SDs $=23 \%$ and $20 \%$; $\operatorname{ts}(11)=6.3$ and 7.2 , each $\mathrm{p}<.01]$. Conditions CD-As expected, when the two types of control contact (control/moving vs. control/ stationary) were paired, there was no difference [means $=42 \%$ and $52 \%$; SDs $=25 \%$ and $17 \%$; ts $(11)=$ 1.2 and .4, each $\mathrm{p}>.05$ ]. Conditions $\mathrm{AC}-$ There was no difference when active touch was compared with control/stationary, the only outcome among the six comparisons not in keeping with expectation [means = $56 \%$ and $50 \%$, SDs $=22 \%$ and $15 \% ; \mathrm{t}(11)=1.0$ and .0 , each $\mathrm{p}>.05]$.

\section{EXPERIMENT 2}

In Experiment 1, the possibility that directionality of action of touch affects apparent arm length was assessed while keeping touch conditions constant (i.e., all six conditions involved touch). The role of directionality of touch vs. the role of contact (touch vs. no touch) was explored in Experiment 2. This was done by use of three conditions: no touch, active touch, and passive touch. If articulation of the body boundary was an effective variable, a decrease in apparent arm length would be expected for both active and passive touch (both involve body boundary contact) as compared with 
the no-touch (control) condition. However, if, relative to contact, directionality of action is the effective variable, then judged arm length should be greater with active touch than with passive touch, with the no-touch control condition falling between.

\section{Method}

A luminous indicator $(5.5 \mathrm{~cm}$ high, $.7 \mathrm{~cm}$ wide) was mounted on the apparatus used in Experiment 1. With eyes open in a dark room, the subject's experienced location of the fingertips of the partially outstretched right arm was measured as follows: The indicator was moved in approximately $1 \leftrightharpoons \mathrm{cm}$ steps toward or away from the subject (in the plane defined by the longitudinal midaxis of the body) until it appeared directly over the fingertips (point of contact of the fingertips with the disk) in the pretest and no-touch conditions (active touch and passive touch conditions). Indicator location could be read to the nearest $1 \mathrm{~mm}$.

In the pretest, one ascending trial (indicator initially $20 \mathrm{~cm}$ closer to the subject than his fingertips) and one descending trial (indicator initially $20 \mathrm{~cm}$ further from the subject than his fingertips) were used. Half the subjects received an ascending trial first. Each subject's own subjective zero regarding fingertip location was computed for each trial by comparing (1) the placement of the indicator when the subject said it was above fingertips and (2) the actual position of the fingertips at the beginning and end of each trial. Deviations of subjective zero away from the subject were arbitrarily coded "+"; those toward the subject were coded "-.."

The point of subjective equality (PSE) of the two pretest trials served as the initial position of the indicator during each of the six subsequent experimental trials (two trials for each of the three experimental conditions). The two trials for each experimental condition were presented consecutively. On each trial, the indicator was placed initially at each subject's "subjective zero" point. Each of the six possible sequences in which three conditions can be presented was employed, with two subjects in each sequence.

Active touch and passive touch were identical to these types of tactual contact used in Experiment 1. For no touch, the outstretched arm of the subject was in the same position as in the active touch and passive touch trials (with the fingertips spread apart and vertically oriented in a frontoparallel plane), but the fingertips did not touch the disk. This was the same physical position of the hand used in the present trials. (The pretest served solely to provide the subjective zero value used as the initial position of the indicator on all subsequent trials, whereas the no-touch trials constituted the comparison control condition.)

The measure of judged arm length was the deviation of the location of the indicator at the end of each trial from each subject's baseline zero. Twelve male college students served as subjects.

\section{Results}

The means for the three main experimental conditions differed, with judged arm longest for active touch (mean $=+2.1 \mathrm{~cm}, \mathrm{SD}=5.92$ ) and shortest for passive touch (mean $=-2.4 \mathrm{~cm}, \mathrm{SD}=5.82)$, and with no touch (mean $=-1.7 \mathrm{~cm}, \mathrm{SD}=5.13)$ falling between $[\mathrm{F}(2,12)=$ $9.41, \mathrm{p}<.01]$. A Newman-Keuls test comparing the means of the three conditions showed that active touch differed from passive touch and from no touch $(p<.01)$, but the latter two conditions did not differ from each other.

\section{DISCUSSION}

The converging evidence from these two studies, each with a somewhat different methodology, is highly consistent with our hypothesis that apparent arm length shrinks when an object acts on the passive hand and increases when the hand acts on an external, stationary object. Specifically, (1) the arm is judged as extending longer under active touch than under passive touch (Experiments 1A, 1B, and 2), (2) the arm with active touch is judged as extending longer than under certain control conditions (control/moving in Experiments 1A and 1B, no touch in Experiment 2) but not all types of control conditions (control/ stationary in Experiments $1 \mathrm{~A}$ and $1 \mathrm{~B}$ ), and (3) the arm with passive touch is judged as extending shorter under certain control conditions (control/stationary and control/moving in Experiments $1 \mathrm{~A}$ and $1 \mathrm{~B}$ ) but not all types of control conditions (no touch in Experiment 2). These findings are consistent with the view that perception is both quantitatively and qualitatively different under active vs. passive conditions (e.g., Gordon, 1978).

How might these findings be interpreted? Consider the body boundary formulation discussed earlier (Humphries, 1959; Wapner et al., 1958, 1963; Werner et al., 1957). According to this view, when a body boundary is touched, there is a decrease in perceived size of the body part. Therefore, if a body boundary process were the main one operative here, both active and passive touch conditions should show shorter apparent arm length than is observed under a no-touch condition. Since judged arm extension was significantly longer for active touch than for no touch, the body boundary interpretation must be questioned.

Can proprioceptive input associated with body motion account for these findings? Three of the six conditions used in Experiment 1 were designed to provide a test between our directionality-of-action hypothesis and a motion hypothesis. These conditions were control/moving vs. control/stationary, active touch vs. control/moving, and control/stationary vs. passive touch. (In the other three conditions, both processes lead to the same prediction.) The findings for the three conditions just specified are in keeping only with the directionality hypothesis. Specifically, for control/moving vs. control/ stationary, the former type of contact involves motion; the latter does not. Based on Fuhrer and Cowan's (1967) finding that vigorous gross body movement resulted in increased size of body parts, a motion hypothesis would predict greater judged arm extension, therefore, for control/moving. The directionalityof-action hypothesis predicted no difference, and no difference was observed. For active touch vs. control/moving, both types of contact equally involve arm-hand motion; a motion hypothesis would thus predict no difference. As for control/stationary vs. passive touch, both types of contact equally involve no motion, again, a motion hypothesis would predict no difference. The directionality-of-action hypothesis predicted that greater judged arm extension would occur with active touch and control/ stationary, respectively, and this is precisely what was observed.

The findings of both studies appear to be entirely in keeping with the direction-of-action hypothesis. When a person stretches his arm out to touch an object, the focus of that action is outward, away from the toucher's body, and this is assumed to be correlated with an increase in judged arm length. In contrast, when the outstretched arm of a person is touched by some other object or person, the focus of that action is inward, toward the body of the person being touched, and this is presumably correlated with judged decrease in length of the extended arm. Our findings and interpretation are in keeping with the speculative phenomenological analysis of Merleau-Ponty (1962), who discusses "being touched" as a passive experience and "touching" as an active experience (i.e., "being impinged upon" and "projecting one's self upon the environment," respectively). 


\section{REFERENCE NOTES}

1. Frey, C. L. Tactual illusions. Paper presented at the annual meeting of the Eastern Psychological Association, Boston, 1967.

2. Frey, C. L., \& Craven, R. A developmental examination of visual and of active and passive tactual horizontal-vertical illusions. Paper presented at the annual meeting of the Eastern Psychological Association, Philadelphia, 1969.

\section{REFERENCES}

Fuhrer, M. J., \& Cowan, C. O. Influrence of active movements, illumination, and sex on estimates of body part size. Perceptual and Motor Skills, 1967, 24, 979-985.

Gibson, J. J. Observations on active touch. Psychological Review, $1962,69,477-490$.

Gordon G. (Ed.). Active touch: The mechanism of recognition of objects by manipulation. Oxford: Pergamon Press, 1978.

HUMPHRIES, O. Effect of articulation of fingertip through touch on apparent length of outstretched arm. Unpublished master's thesis, Clark University, 1959.
Merleau-Ponty, M. Phenomenology of perception. London: Routledge \& Kegan Paul, 1962.

WAPNER, S. Organismic-developmental theory: Some applications to cognition. In P. H. Mussen, J. Langer, \& M. Covington (Eds.), Trends and issues in developmental psychology. New York: Holt, Rinehart, \& Winston, 1969.

WapNer, S., McFarland, J. H., \& Werner, H. Effect of visual spatial context on perception of one's own body. British Journal of Psychology, 1963, 54, 41-49.

Wapner, S., Werner, H., \& Comalli, P. E. Effect of enhancement of head boundary on head size and shape. Perceptual and Motor Skills, 1958, 8, 319-325.

WeRNER, H. Comparative psychology of mental development. New York: Harper, 1940.

Werner, H., Wapner, S., \& Comalli, P. E. Effect of boundary on perception of head size. Perceptual and Motor Skills, 1957, 7, 69-71.

(Received for publication August 4, 1981.) 